\title{
Selfish Node Detection for Effective Data Transmission Using Modified Incentive Sorted Pathway Selection in Wireless Sensor Networks
}

\author{
P. Ashok Babu, ${ }^{1}$ L. Kavisankar, ${ }^{2}$ Jasmine Xavier, ${ }^{3}$ V. Senthilkumar, ${ }^{4}$ Gokul Kumar, ${ }^{5}$ \\ T. Kavitha, ${ }^{6}$ A. Rajendran, ${ }^{7}$ G. Harikrishnan, ${ }^{8}$ A. Rajaram, ${ }^{9}$ and Amsalu Gosu Adigo ${ }^{10}$ \\ ${ }^{1}$ Department of Electronics and Communication Engineering, Institute of Aeronautical Engineering, Dundigal, Hyderabad, India \\ ${ }^{2}$ Department of Computer Science and Engineering, SRM Institute of Science and Technology, Chennai, India \\ ${ }^{3}$ St.Xavier's College (Autonomous), Palayamkottai, Tamil Nadu, India \\ ${ }^{4}$ Department of Electronics and Communication Engineering, Megha Institute of Engineering and Technology, Ghatkesar, India \\ ${ }^{5}$ Manager Technical Supply Chain, Rossell Techsys, Bangalore 562129, India \\ ${ }^{6}$ Department of Computer Applications, Kongu Engineering College, Perundurai, India \\ ${ }^{7}$ Department Electronics and Communication Engineering, Karpagam College of Engineering, Othakalmandapam, \\ Coimbatore, India \\ ${ }^{8}$ Holy Grace Academy of Engineering, Mala, Thrissur, Kerala 680732, India \\ ${ }^{9}$ Department of Electronics and Communication Engineering, EGS Pillay Engineering College, Nagapattinam 611002, India \\ ${ }^{10}$ Center of Excellence for Bioprocess and Biotechnology, \\ Department of Chemical Engineering College of Biological and Chemical Engineering, \\ Addis Ababa Science and Technology University, Addis Ababa, Ethiopia
}

Correspondence should be addressed to Amsalu Gosu Adigo; amsalu.gosu@aastu.edu.et

Received 18 December 2021; Revised 12 January 2022; Accepted 17 January 2022; Published 18 February 2022

Academic Editor: Deepak Kumar Jain

Copyright $\odot 2022$ P. Ashok Babu et al. This is an open access article distributed under the Creative Commons Attribution License, which permits unrestricted use, distribution, and reproduction in any medium, provided the original work is properly cited.

\begin{abstract}
Wireless nodes have time-varying behavior in their nature because the energy usage of each node is not being constant. Packet transmission among cluster nodes gets overloaded when multi-input and multioutput is made; multitask is performed causing more energy usage; also, selfish node could not transfer the data packet to the target node. Selfish nodes need to hide some important data and then only transmit normal data that try to misuse those data, so packet delivery rate is reduced. This normal method does not identify selfish nodes in the routing path. Proposed Incentive Sorted Path Selection Scheme (ISPSS), the progressively stable algorithm, enables the identification of such selfish nodes. The results confirm the enhancement in the lifetime of the network, the energy efficiency, and the delivery rates of the packets with reduced end-to-end delay.
\end{abstract}

\section{Introduction}

The latest techniques move forwards the arrangement of little low-cost sensor nodes against a section of concentration to organize position information, to provide a route for packet sharing with also a path to a sink node for packet organization, that in total contain a sensor network [1]. It is used in several uses in temperature analyzing, adversity managing, record analyzing, smart spaces, exactness gardening, end monitoring, and observation. In a multihop wireless sensor network with a permanent overloading state, every sensor node functions as an information collector node that generates data packets to the network at a nominal time gap, to send its data packets to the base station sink node [2].

At a similar time, all nodes should work as transmitters to broadcast the remaining data packets to the base station node through various routes. In different uses, the sensor 
nodes have more energy and lack any renewing service [3]. Mainly of the sensor nodes energy level is low in the communication performed in network environment. While the energy level is worn out, a node fails to work and predictably this stops the lifespan of the network that shows the time period of communication until the drop in initial node, mostly because of the lacking energy in the network [4]. Consequently, for the efficient energy use, the predominant thing to be minimized is the entire quantity of packet communication in sensor environment.

Packet overload is total amount of packets should be reached into the sink node is lesser surrounded by the reporting restriction [5]. Those clear, it is evident that all packet must follow the route with lesser hop to minimize the total quantity of communications. Except if nodes forever broadcast their packets to the base station node through minimum hop routes, nodes quicker to the base station should take more load and will drain their energy sooner, generating energy holes [6] in the order of the base station node.

Therefore, in the wireless multihop sensor network, the demanding problem is to use the energy or power of all the nodes consistently to enhance the lifespan of the entire network [7]. Data collecting with traffic management in conditions of energy broadcast at each entity node may be a well-organized method to increase the network lifespan. It should report newly on this problem. But in most of the previous processes, load evaluation should be obtained at the expenditure of communicating through more distance path that is not the best from energy direction of vision [8]. Here, nodes take a choice to broadcast data packets more overstraight through different routes direct to assurance energy management.

The higher and lesser limits on well-designed lifespan are derived. Heuristic method with communication energy control ensures higher network lifespan by managing overhead communication as equally as probable [9]. Those methods traffic management is besieged at the charge of improved energy usage in communication among inefficient paths. Normal conditions for the correct allocation of the base station node, else intermediate nodes, are used. For using more data association, transmitting with data combination [10] has emerged as a helpful concept for traffic management which should not decide the energy hole issues completely. At the same time, it needs multifaceted calculation to avoid defeat of very important data.

This obtains traffic-free packet transmission among wireless sensor nodes. The routing paths are sorted based on their distance and energy usage. Thus, the effective pathway for transmission from the source node to the base sink node utilizes relatively less energy and is at a shorter distance. The selfish node exists in the clusters.

From here the paper is discussed in sections as given below. Section 2 discusses the related previous works. In Section 3, the details of the proposed Modified Incentive Sorted Pathway Selection Approach (ISPS) are discussed to opt for an efficient way for the communication in the wireless sensor network (WSN) technology. It also deals with the identification and deletion of the selfish nodes present in the clusters. Section 4 provides detailed insights to carry the simulation and analysis of the results to examine the performance obtained with respect to various parametric conditions. Finally, Section 5 concludes the proposed work and also elaborates an idea for future work.

\section{Previous Works}

Naeem, Muhammad, and team [11] presented an understanding through group of Multiple Input Multiple Output path; also continuation of a reply path among the groups with fusion center is the input of the present environment. The energetic characteristics should bead opt within the environment are present guide copied from the accepted analyzing of the path superiority that has been attain among the reply path from the mixture center. The energetic possessions of the present environment make it strong over time, adjusting character of the spread infrastructure. Network is self-governing of the character of the analyzing use, obtained with general character. When compared to the existing DDEEC and EDDEEC methods, the experimental output of the clustering method has the longest network lifetime. In addition, it is validated by output of proposed framework obtaining a trade-off model among network life span with communication trust worthiness.

Chatterjee et al. [12] present a method which creates lesser overload, enough for reporting. Depending on this node sharing, a dispersed, almost traffic managing data collecting scheme is launched for packets to reach the base station through less hop routes that also in turn supports boundary of the overload. A standard case probabilistic investigation is made which depends on great identical random links to find an imaginary lesser hop on the amount of nodes to be arranged. Output indicates the proposed model has higher lifespan of network and minimum cost routing path compared to previous methods.

Feng et al. [13] proposed adaptive packet broadcasting in sensor network afflicted with possible selfish nodes. Well Suited ICMP motivation is more copy of information. Allow for the rules that node's broadcasting decision in this method damaged by its present energy level; with network infrastructure it is difficult to share data through nodes to guide their broadcasting characters. In the meantime, the broadcasting character updates create the evolutionary stable method for this environment. It reduces error and improves accuracy of packet transmission.

Nithyakalyani and Suresh Kumar [14] present various analyzing methods that suggest various group head choice methods for data collection. This method studies the data relay K-means grouping method, with unclear grouping methods with inherited grouping technique. Important factors for estimating and distinguishing these algorithms are distinct, characterized, and conclude. To consider a thought that the sensor nodes are aimlessly dispersed and are not fixed, data packets organization of the sink node with the size of the sensor ground is identified.

Dey et al. [15] proposed work on constructing a grouping method stimulated from Fruit Fly association to optimize energy efficiency of the nodes in the sensor network with reduced pathway route for the communication. Also, they 
are involved in improving the lifespan of nodes since each node has more energy. The overall network is partitioned with various ranges by the Fruit Fly model. The primary effectiveness of energy usage is essential to design the FF method. Lifespan of network is measured based on cluster head chosen scheme with data transmission effectiveness being analyzed depending on groups in network. It easy to find misroute data packet compared to previous method.

Justus and Chandra Sekar [16] presented that many routing techniques are used to find and remove blocking in sensor network. For traffic management in sensor network, there is want to place and find routing Data packets concerning all node in the network for Better communication route discovering from source node to target node in network. Path Selection approach is altered by the DalPaS Hybridized Dynamic for the traffic management in the sensor networks. The collected packets are done with the nodes that exist on the network. For reduced overload, routing procedure is carried out. Better transmission rate is achieved in present method compared to existing method.

Kurt et al. [17] proposed broadcast energy task with packet size chosen to be consistent. Consequently, joint analyzing of communication energy also packet size is of greatest vital one in sensor network lifespan improvement. To design a complete LL-link layer model, the behavior of the sky network sensor nodes and their path depends on the normal analyzing with route failure of different infrastructures. A novel MIP-Mixed Integer training generates support for the link layer model to enhance sensor's lifespan by the joint optimization of communication energy range with data packet size. It obtains network infrastructure through the numerical results of the optimization scheme.

Celik and Kamal [18] proposed effective cluster head that provides the lesser collision rate in communication and best routing routes from sender to target node. Exploit PoissonBinomial sharing, widespread $\mathrm{K}$-out-of $\mathrm{N}$ voting condition is launched for heterogeneous nodes to accept sender to have various normal identification of collision. This convex optimization method is well-known to reduce the intracluster energy usage by jointly obtaining the better sensing times and limits of feature identifiers for this condition. A common fixed sample size examination is done to create a weighted sample size survey for quantized flexible decision combination to achieve a more energetic communication. It is better one for cluster head chosen compared to existing methods.

Gong et al. [19] proposed WARAS-adaptive path chosen motivated by metabolism characters of Escherichia coli. It contains of two things; the first one is a path activity model to show alteration integrity of multipath overload in communication to normal network, that is in reverse relative to total dissimilarity among current route capacity and better route capacity. Next are multiattractor rules reconstructed by specifying important effects of congestion on the route chosen. It is easy for present scheme to find congestion compared with lots of attack identification methods. It performs smooth communication so minimum energy is used.
Li et al. [20] proposed an RNs' NSI which contains DeN (degree of node) intrinsic selfishness and extrinsic selfishness. Below the disseminated node self-interest control, a route chosen is constructed to choose the most effective and minimum distance routes that cause more energy usage. Present method finds the misbehaving node and source broadcast data packet to target node in best route. Experimental output manages the selfish node behavior and provides effective communication in wireless sensor network environment.

Gong et al. [21] presented scheme adaptive path chosen scheme which is also known as WSN path chosen scheme depending on the adaptive response by characteristics selection model that is inspired.

Mohanty and Kabat [22] presented a method to keep away from congested node traffics with the calculation of the possibilities for jamming in the nodes, relay nodes, speedy transmission, and the data packet broadcast range in the relaying node that is known. The storage limit at each node is the distribution to hold for the reasonable and prominent data rate successes.

Premanand and Rajaram [23] present an improved architecture for solving data inaccuracy problem in route selection. Due to the dynamic nature of mobile nodes, optimum path selection for enhancing the data transmission ratio is a challenging task. To address this issue, higher accuracy is achieved through the new routing protocol. This is executed for every successful and unsuccessful data communication.

Palaniswami and Rajaram [24] propose the malicious node detection technique for MANET environment via managing the trustworthiness of each mobile user in the network. For that, trust value of mobile node is computed and added in the packet header. Based on the information, packet forwarding node is selected and also malicious node is detected.

Palaniswamy and Rajaram [25] propose a security architecture for providing the data integrity, confidentiality, and authentication features to detect and mitigate the security attacks. For security improvement, Shamir's Secret Sharing is proposed for two operations such as Certificate Revocation and Certificate Renewal at the Certificate Authority.

\section{Overview of Proposed Model}

Wireless sensor nodes perform packet transmission in cluster, but traffic occurred when packet transmission is delayed by selfish node that is present in routing path. There is selfish node available in routing path, it hides the vital information from packet, and it forwards normal data to next neighbor node in routing path to sink node. Incentive Sorted Path Selection Scheme is present to control the packet overload for each transmission. Progressive stable algorithm is implemented to detect the selfish node, depending on behavior that node adjusts the frequency level from low to high; in that time packet information is hidden by selfish node. 
Figure 1 shows the Modified Incentive Sorted Pathway Selection Model. The nodes that act as the cluster head are involved in coordination with all other members in the network. It performs the collection of information of the entire nodes present in that particular cluster. Modified Incentive Sorted Pathway selection model is proposed to identify the traffic rates in the network. This is done by implementing the progressively stable algorithm concept. This algorithm periodically monitors the node frequency updates and encounters the selfish node. For instance, if there exists no traffic in the network, it indicates that there is the possibility for smooth transmission in the path. Thus, it enhanced delivery rate of the packets and minimized traffic or overhead of communication.

The nodes of the self-directed transmitting packets are suffered due to selfishness. Pointing to the selfishness of any particular node that exists in the network environment is considered to merit, irrespective of the broadcasting data packet construction; it also controls the protection issues. In the WSN communication process, two different types of selfishness are analyzed, they are the entity and the common methods. The detection of single selfishness denotes the irregularity in the node network that concentrates on their own security reasons. Also, the selection of the selfish nodes supports the remaining members.

3.1. Cluster Heads Coordinate Their Member. A preparation of sensor nodes into various effective clusters is called grouping. Each group is comprised of a cluster head and its fellow member nodes. The functionality of the cluster head is to monitor and manage the member nodes in the group and perform intercluster packet organization and broadcasting of the data packets. They segregate the data packets that are transmitted to the base station node. Thus, reduced traffic is witnessed.

An energetic grouping and the formation of relay method are done for the time-driven and the event-driven purposes. Furthermore, a common network is used for the adaptive operation of the aforesaid analyzing situation to improve its possibility of construction for a dissimilar choice of uses. Additionally, the dynamic portion of the quantity of cooperation depending on path broadcast conditions is also measured. The structured sensor network selects the decisions, the choice of the cluster head, organizing groups with its nodes intermediate, and the option of sensor nodes support for the coverage to the center combination which happened within the individual group's transmission range in the network. $C_{o}$ is the coordination of cluster coordination and it is expressed as

$$
C_{o}=C_{m} *\{\mathrm{DC} \mid \mathrm{DB}\}
$$

$C_{m}$ is cluster member, and $\{\mathrm{DC} \mid \mathrm{DB}\}$ is data collection with data broadcasting. If the networks are individual hop models then each sensor node broadcasts its packets straight to the base station in the network. Those designs do not support the large area because communication cost becomes so costly for energy usage. In the multihop flat scheme, sensor nodes broadcast similar data packets such as routing tables. Network overhead and energy usage are improved, and usage of the resource is not to be more. At last in the grouping scheme, sensor nodes have minimum traffic with minimum energy usage due to the data organization scheme being done in cluster head; also cluster head intermediate should aggregate data packets to the base station node. In the grouping method, resources are assigned to all groups to minimize intrusion among cluster members, so network lifetime is improved, and nodes are active for a long period of time. In simulation output, many clustering methods are suitable for the remote large-scale area network.

$$
\begin{aligned}
C_{m} & =\lim _{m} n, \\
\lim _{m} n & =\frac{\sum \csc ^{-1} n}{m} .
\end{aligned}
$$

A dispersed group head chosen method is present in which each of the sensor nodes that can provide the role with lesser energy usage has an option to become head group node. Initially, each of the sensor nodes will transmit information to the cluster heads and finally to the sink node of the network. At first, each of the sensor nodes is normal to estimate their space from the center of its base station node.

3.2. Modified Incentive Sorted Pathway Selection. The sensor nodes are normal to grade depending on their distance from the mid of the group individually. The nearest source node of the mid to the grid has the maximum chance of becoming the group head. The threshold for energy consumption of a particular channel is suspiciously definite, if the energy usage of a cluster head categorizes to down the energy level for each channel. The position of the group report is usually to be broadcast to the next maximum chance node. Previously each cluster head is chosen; the residual nodes discover the nearest cluster head nodes and add them irrespective of their original group obligation. It selects the group header nodes, with the designing of new groups in the network.

$$
\frac{\csc ^{-1} n}{m}=m_{n} \log m \text {. }
$$

Cluster heads carry out more processes for their individual source nodes' performance at efficient energy than the nongroup head node. Popularly used grouping methods perform, revolving the group headeract between sensor nodes when reducing the regularity of regrouping, it is vital to discover the best routing in an efficient manner.

$$
m_{n} \log m=\frac{m_{n} \log m}{S(P)} .
$$

Process for nodes construction is used to discover the better value to describe path that needs extensive experimental output. As the sharing of sensor nodes is normal to be chance in most of the uses, unstable grouping is needed to construct that adjustment with changeable rules within the network. Subsequently, the methods to discover the best limit strength modify transmission rate so the lifetime of the network is reduced since the selfish node is caused to damage packet transmission on the choosing of the routing 


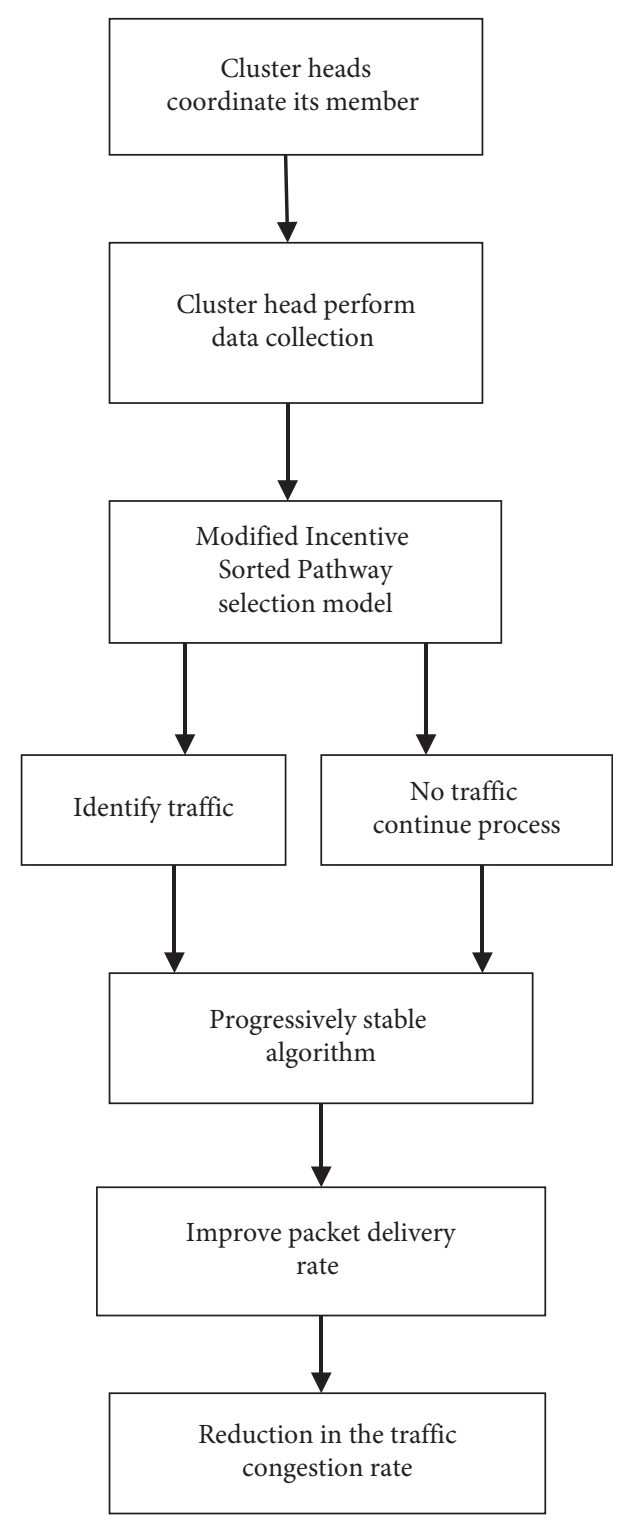

FIGURE 1: Block diagram of the Modified Incentive Sorted Pathway selection model.

path and therefore can cause more energy usage in the network environment. To defeat these boundaries aforesaid limit is fixed for selection technique; a soft decision depends on threshold chosen scheme.

$$
\begin{aligned}
C_{m} & =\frac{m_{n} \log m}{S(P)}, \\
\{\mathrm{DC} \mid \mathrm{DB}\} & =\frac{\hat{\mathrm{e}}(\mathrm{DC})}{\hat{\mathrm{e}}(\mathrm{DB})} .
\end{aligned}
$$

The data creator nodes alter the amount of information collected in a particular routing path. It is an efficient way in which the amount of packet improves; the proportion of router nodes increase quickly with the percentage of data creator nodes should not be important. The minimum amount of information creator nodes limits the quantity of data packets created in the routing path and therefore improves the lifespan of the network considerably. In addition, it is obvious that networks with more packets should reduce traffic occurrence when the selfish node is identified.

$$
\frac{\hat{e}(D C)}{2 \hat{e}(D B)}=\frac{D C}{2 D B} \text {. }
$$

This incentive sorted path choosing method removes the selfish node which presents in the routing path; cluster head collects data packets in a frequent manner. Selfish node disturbance causes data packet transmission traffic to occur. By using ISPS method packet latency is reduced, because it does not allow traffic occurrence in every time slot. The energy level of all nodes in the cluster maintains a similar range, as the delivery rate of the packets increases (Algorithms). Progressively stable routing analyzes each node's behavior if the node varying the frequency level causes loss of data packets during the transmission time period. These nodes are identified and marked to remove from the particular cluster, which is the selfish node for transmission. Initially, it hides some of the essential details and broadcasts the normal details alone to the cluster head and finally to the base sink node (Algorithm 2).

3.3. Progressively Stable Algorithm. The header node quantities are chosen arbitrarily. The header node begins to increase in wraps approximately. During each transmission, the nodes of a wrap choose a single-hop intermediate node, which improves the coverage and broadcasts the data packet to the header. Surrounded by all these nodes header chooses the efficient path that should contain higher coverage range and transmit data packets. All header finishes while its separation outputs the needed coverage; else no linked intermediate node of the divider is gone. Last, headers that are doing well broadcast its separation to the base station node.

$$
\begin{aligned}
C_{o} & =\frac{C_{m} * \mathrm{DC}}{2 \mathrm{DB}}, \\
C_{o} & =\frac{m_{n} \log m}{S(P)} * \frac{\mathrm{DC}}{2 \mathrm{DB}} .
\end{aligned}
$$

It is assured that all information is created by a data creators to effectively attain the base station node through single transmitter nodes in straight data maintaining the network's practical traffic management. The chance of nonuniform node distribution does not assure that great corresponding should exist. By using the progressively stable algorithm, whether a node contains data packets should lose to discover an equal data its packet is should be dropped which should make some region of the neighborhood to stay exposed.

Progressively stable routing analyzes each node's behavior if the node varying the frequency level causes loss of data packets during the transmission time period. Those nodes are identified and marked to remove from the particular cluster, which is the selfish node for transmission. Initially, it hides some of the essential details and broadcasts the normal details alone to the cluster head, and finally to the base sink node.

It enables the Modified Incentive Sorted Pathway for the packet collected by the cluster header node. Progressively 
(1) for every selection of sorted path

(2) if $\{$ path $==$ selfishnode $\}$

(3) ill-treat transmitted data packets

(4) hiding confidential information, broadcasting only the normal information.

(5) thus, identify and discard the selfish node

(6) else,

(7) if $\{$ selfish node $=$ path! $\}$

(8) proceed transmission of packets efficiently

(9) packet data organization by the cluster head

(10) END_if

(11) END_for

Algorithm 1: Modified Incentive Sorted Pathway selection algorithm.
(1) if $\{$ Node $==$ Stablecommunication $\}$
(2) for individual path checked
(3) frequency update analysis results as no change in frequency.
(4) establishment of connections for the packet transmission
(5) forward the collected cluster head to the sink node.
(6) else
(7) if $\{$ node $==$ Unstablecommunication $\}$
(8) frequency update analysis results in a lot of changes in frequency.
(9) selfish node discard in the routing path. Step10: END_for Step 11: END_if

Algorithm 2: Progressively stable algorithm.

stable energy-based routing is obtained, when the efficient nodes are only chosen to perform communication between cluster member node and base station node or sink node. It improves the packet delivery ratio and also minimizes energy and end-to-end delay.

Packet ID: It comprises a whole wireless sensor network node data based on its behavior and node deployment in the network environment.

In Figure 2, the existing packet format is presented. Three bytes are consumed by the source node ID field and three bytes are consumed by the destination node ID field. The third one is cluster heads to coordinate its member to carry 5 bytes. The fourth field occupies 6 bytes. The Modified Incentive Sorted Pathway selection model is applied to clustering; cluster head organizes data packets in an effective routing path. So energy consumption is reduced. The fifth has 3 bytes. Progressive stable algorithm is constructed to improve stable communication and monitor the frequency changes of each node. The final file is the improved packet delivery ratio, and it consumes 4 bytes of data. Thus the identification and removal of selfish nodes are done in the routing path.

\section{Performance Analysis}

4.1. Experimentation. The projected Internet Services Provider's model is simulated using the NS 2.34, Network Simulator tool. The simulation parameter includes 100 sensor nodes employed in the area with 1060 meters $\times 930$ meters square' region for about 80 milliseconds of simulation time. All the sensor nodes were organized in a haphazard manner within the network. These nodes exhibit the same transmission range of around 250 meters. CBR (Constant Bit Rate) delivers a constant speed to the transmission of packets in the network, thus contributing to limiting the rate of traffic occurrence. DSR (Dynamic source routing) protocol offers an effective communication pathway for the transmission of the packets in the clustered phase and it also identifies the selfish nodes in the routing pathway. Table 1 gives the parameters used for the initialization of the NS.

\subsubsection{Simulation Results. 4.1.2. Simulation Outcome.} Figure 3 depicts the projected Internet Service Providers method enabling the traffic-free packet transmission among cluster nodes in sensor network; it chooses an efficient routing path compared to the existing Universal Dynamic Clustering Scheme approach [11] and the Leaky Bucket Counter approach [12]. Projected Internet Service Providers method with the progressively stable routing algorithm is used for the stabilization of the node energy during the routing process and detects the selfish node during transmission time, so it increases packet delivery rate and minimizes delay.

4.2. Performance Analysis. To analyze the performance of the network, the simulation was carried out using the parameter X graph in the network simulator 2.34. 


\begin{tabular}{|c|c|}
\hline The Source ID & 3 \\
\hline The DestinationID & 3 \\
\hline Improved data packetdelivery ratio & 4 \\
\hline Cluster headscoordinate their member nodes & 5 \\
\hline Progressively stable algorithm & 3 \\
\hline Modified Incentive Sorted Pathway selection & 6 \\
\hline
\end{tabular}

FIGURE 2: The projected internet service provider's packet format.

TABLE 1: Experimental parameter setup.

Number of nodes used

Area dimensions

Mac address used

Radio coverage range

The time limit used for simulation

Source traffic used

The size of the packets

Agility model

Procedure
100 No.

$1060 \mathrm{~m} \times 930 \mathrm{~m}$

IP-802.11 g

$250 \mathrm{~m}$

$30 \mathrm{~ms}$

CBR

150 bytes

Random way point

Dynamic source routing protocol

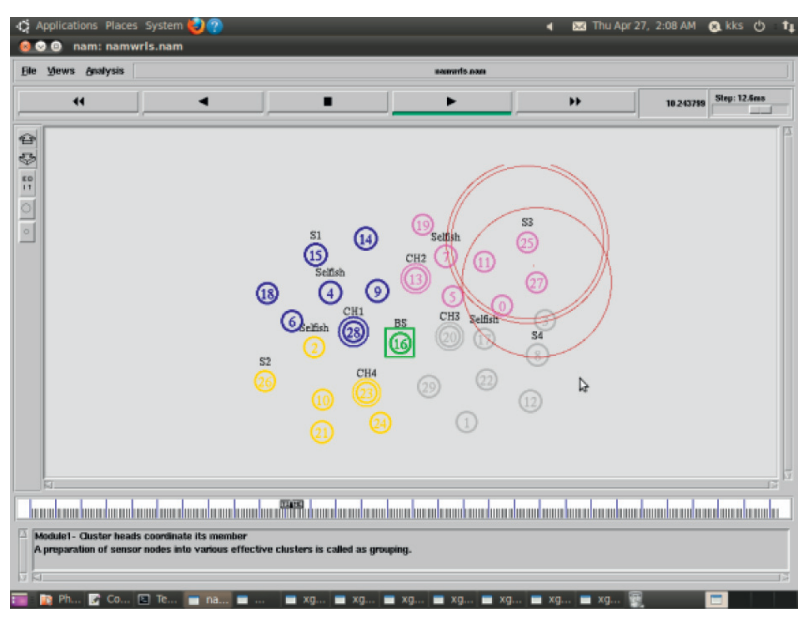

Figure 3: Projected Internet service provider's simulation result.

4.2.1. End-to-End Delay. From Figure 4 the end-to-end delay is evaluated by using the time slot used for communication between the sender and the cluster head node, with progressive stable routing support to remove the selfish attack. In the proposed Internet Service Providers method the end-to-end delay is reduced comparatively.

$$
\text { end to end delay }=\text { end time-start time. }
$$

4.2.2. Communication Overhead. Figure 5 confirms that the communication overhead is minimized as the cluster head node organizes the data packet with an efficient network connection. The data packets are efficiently acknowledged by the cluster head. In the proposed Internet Service Providers scheme the communication overhead is reduced comparatively compared to the existing methods like Leaky Bucket Counter protocol, NARP, LORP, Universal Dynamic Clustering, EPCSA, etc.

$$
\text { communication overhead }=\left(\frac{\text { number of packet losses }}{\text { received }}\right) * 100 \text {. }
$$

4.2.3. The Delivery Ratio of the Packets. Figure 6 depicts the packet delivery ratio which is measured by the amount of data packets received from the amount of data packets sent. Generally, the transmission rate was set as 100 , and the trustworthy node connection was recognized to achieve an efficient data packet organization by the cluster head and the base station node. Thus the work presented showed an increased packet delivery ratio compared to all other existing approaches.

packet delivery ratio $=\left(\frac{\text { number of packets received }}{\text { sent }}\right) * 100$.

4.2.4. The Lifetime of the Network. Figure 7 depicts that the lifetime of the network is determined by the performance of the entire network, the amount of energy, and the time required for the transmission. Stable energy nodes are used for the routing process; also, the selfishness nodes were identified and removed. In the proposed ISP's method, the network lifetime was found to be increased more than in the other existing approaches like LBC, RCDA, TDTC, LORP, UDCS, etc. 


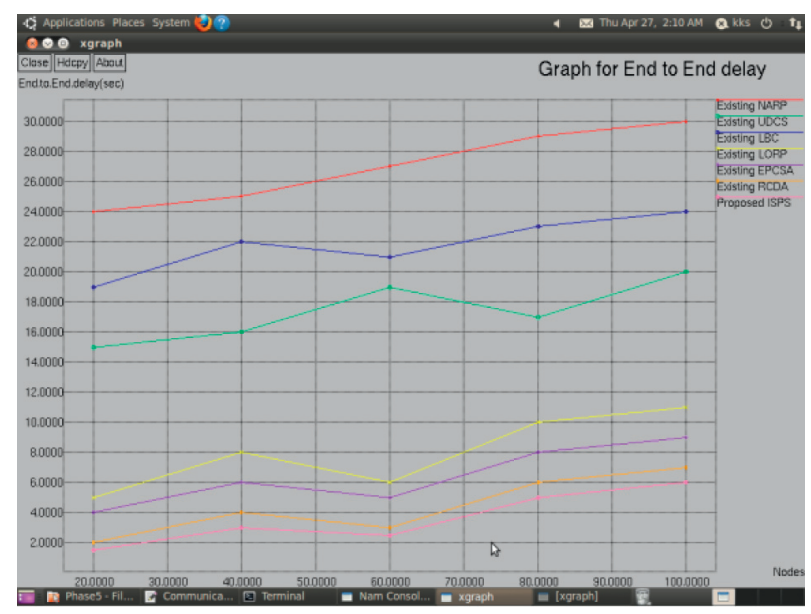

FIGURE 4: Graphical result for the nodes vs. the end-to-end delay.

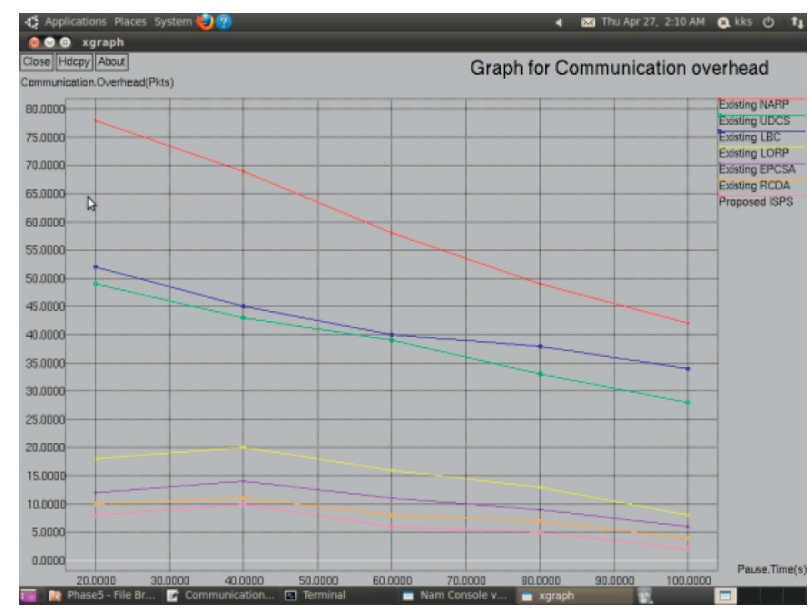

FIGURE 5: Graphical result for the pause time vs. the communication overhead.

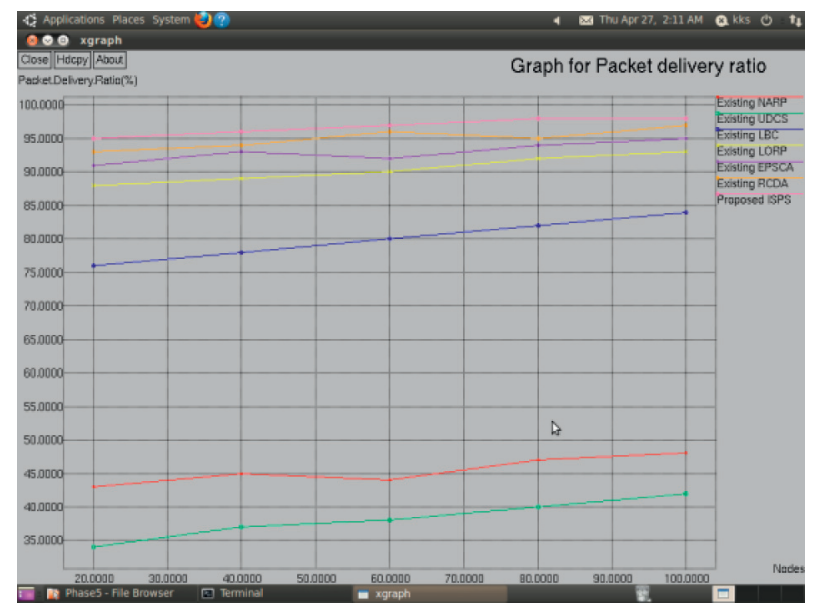

Figure 6: The graphical result for the nodes vs. the packet delivery ratio. 


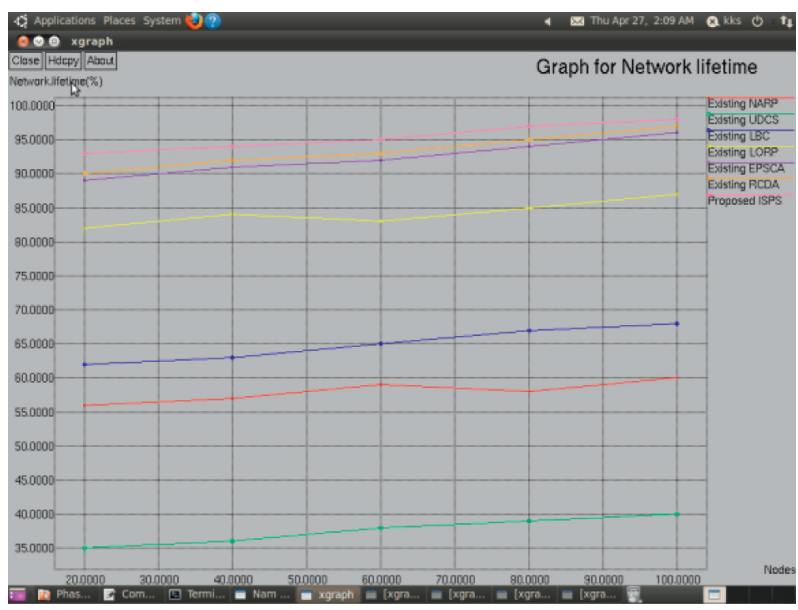

FIgURE 7: The graphical result for the nodes vs. the network lifetime.

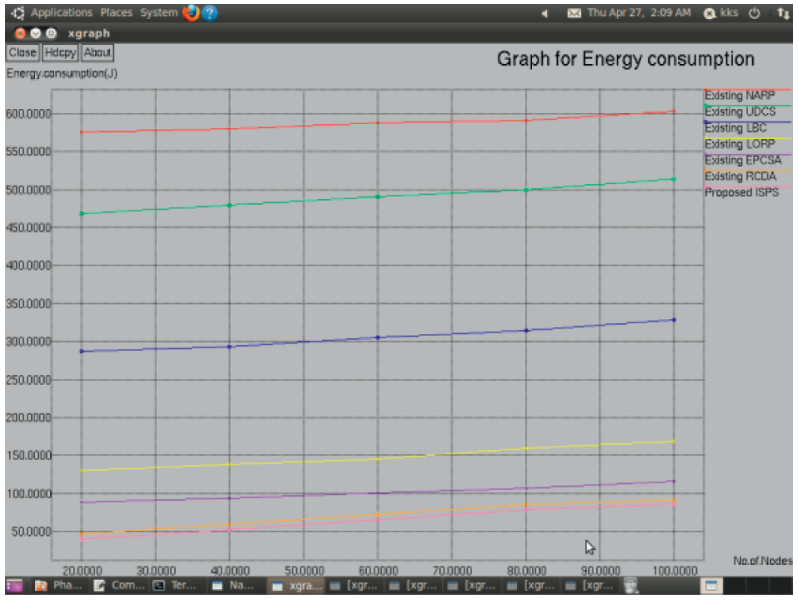

Figure 8: The graphical result for the number of nodes vs. the energy consumption.

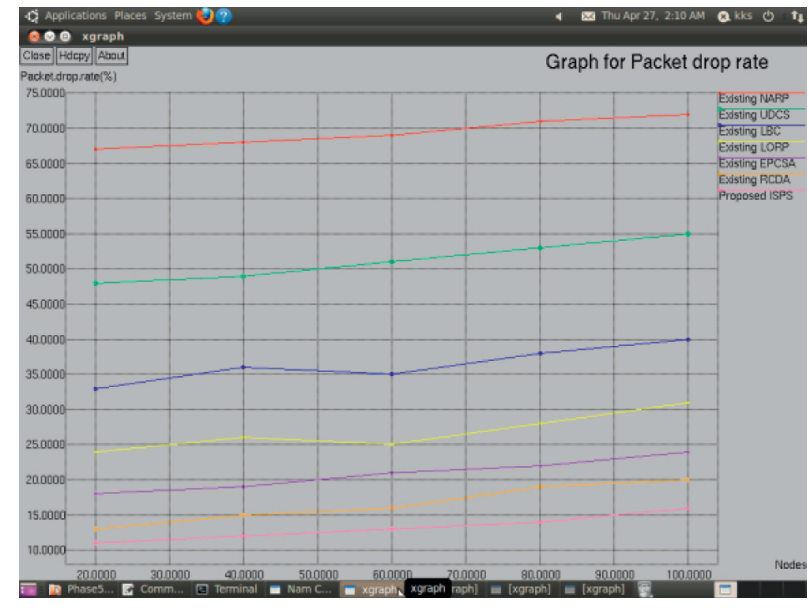

FIgURE 9: The graphical result for the nodes vs. the packet drop rate. 


$$
\text { network lifetime }=\frac{\text { length of energy usage }}{\text { overall energy }} \text {. }
$$

4.2.5. Consumption of Energy. Figure 8 depicts the energy consumption, which is the amount of energy spent for the transmission of data from the starting node to the ending node. In the projected model the analysis of the frequency in the nodes and frequency updates were done by using the progressively stable routing algorithm. The energy consumption is minimized more than that of the other existing protocols.

$$
\text { energy consumption }=\frac{\text { initial energy }}{\text { final energy }} \text {. }
$$

4.2.6. Packet Drop Rate. Figure 9 represents that the loss of the data packets was reduced; it eliminates the communication link within the sensor nodes of the cluster. It also helps in the removal of the selfish node which was made available in the routing pathway. In the proposed ISPS model, the drop rates of the packet are reduced compared to the other existing methods.

$$
\text { packet drop rate }=\left(\text { number of packets } \frac{\text { dropped }}{\text { sent }}\right) * 100 \text {. }
$$

\section{Conclusion}

All sensor nodes need to perform multitask at the same time that causes the depletion of energy in the network so that the link quality is not sufficient. The selfish nodes in the network misuse the essential information and only forward the important information to the next neighbor node in the cluster. And so the packet drop occurs. This proposed ISPS method establishes the routing path way as the traffic-free relay nodes in the cluster are not the best and have selfish behavior. This selfish node distributes all the information to the unwanted or out-ofcoverage nodes, and so more energy is used. Thus, they are identified and removed from the entire network environment. Progressively stable routing algorithm promotes the frequency analysis updates during the transmission, it is easy for it to find the selfish node, and it also improves the packet delivery ratio and reduces the end-to-end delay process. Further in the future, the focus of boundary coverage monitoring for the analysis of various metrics in the sensor network can be carried out.

\section{Data Availability}

The datasets used and/or analyzed during the current study are available from the corresponding author on reasonable request.

\section{Conflicts of Interest}

There are no conflicts of interest.

\section{References}

[1] S. K. A. Imon, A. Khan, M. D. Francesco, and S. K. Das, "RaSMaLai: a randomized switching algorithm for maximizing lifetime in treebased wireless sensor networks," in Proceedings of the IEEE INFOCOM, pp. 2913-2921, Turin, Italy, April 2013.

[2] R. Kacimi, R. Dhaou, and A.-L. Beylot, "Load balancing techniques for lifetime maximizing in wireless sensor networks," Ad Hoc Networks, vol. 11, no. 8, pp. 2172-2186, 2013.

[3] A. Jarry, P. Leone, O. Powell, and J. Rolim, "An optimal data propagation algorithm for maximizing the lifespan of sensor networks," in Proceedings of the IEEE International Conference on Distributed Computing in Sensor Systems, pp. 405-421, San Francisco, USA, June 2006.

[4] A. Jarry, P. Leone, S. Nikoletseas, and J. Rolim, "Optimal data gathering paths and energy-balance mechanisms in wireless networks," Ad Hoc Networks, vol. 9, no. 6, pp. 1036-1048, 2011.

[5] J. C. Dagher, M. W. Marcellin, and M. A. Neifeld, "A theory for maximizing the lifetime of sensor networks," IEEE Transactions on Communications, vol. 55, no. 2, pp. 323-332, 2007.

[6] C. Efthymiou, S. E. Nikoletseas, and J. D. P. Rolim, "Energy balanced data propagation in wireless sensor networks," Wireless Networks, vol. 12, no. 6, pp. 691-707, 2006.

[7] A. Giridhar and P. R. Kumar, "Maximizing the functional lifetime of sensor networks," in Proceedings of the 4th international symposium on Information processing in sensor networks, pp. 5-12, Los Angeles, California, January 2005.

[8] P. Casari, M. Nati, C. Petrioli, and M. Zorzi, "Alba: An adaptive load-balanced algorithm for geographic forwarding in wireless sensor networks," in Proceedings of the IEEE MILCOM, pp. 1-9, Washington, DC, USA, October 2006.

[9] S. C. Ergen and P. Varaiya, "Optimal placement of relay nodes for energy efficiency in sensor networks," in Proceedings of the IEEE International Conference on Communications (ICC), pp. 3473-3479, Istanbul, Turkey, June 2006.

[10] S. Olariu and I. Stojmenovic, "Design guidelines for maximizing lifetime and avoiding energy holes in sensor networks with uniform distribution and uniform reporting," in Proceedings of the IEEE INFOCOM, pp. 1-12, Barcelona, Spain, April 2006.

[11] M. Naeem, M. Patwary, and M. Abdel-Maguid, "Universal and dynamic clustering scheme for energy constrained cooperative wireless sensor networks," IEEE Access, vol. 5, 2017.

[12] P. Chatterjee, S. C. Ghosh, and N. Das, "Load balanced coverage with graded node deployment in wireless sensor networks," IEEE Transactions on Multi-Scale Computing Systems, vol. 3, 2017.

[13] Li Feng, Q. Yang, and K.-sup Kwak, "Incentive-compatible packet forwarding in mobile social networks via evolutionary game theory," IEEE Access, vol. 5, 2017.

[14] S. Nithyakalyani and S. Suresh Kumar, "Data aggregation in wireless sensor network using node clustering algorithms-a comparative study," in Proceedings of the 2013 IEEE Conference on Information \& Communication Technologies (ICT), IEEE, Thuckalay, India, April 2013.

[15] A. Dey, T. Sarkar, and S. Ali, "Fruit Fly algorithm based clustering protocol in wireless sensor networks," in Proceedings of the 2016 9th International Conference on Electrical and Computer Engineering (ICECE), December 2016.

[16] J. J. Justus and A. Chandra Sekar, "Congestion control in wireless sensor network using hybrid epidermic and DAIPaS 
approach," in Proceedings of the International Conference on Inventive Computation Technologies (ICICT), vol. 3, August 2016.

[17] S. Kurt, H. U. Yildiz, M. Yigit, B. Tavli, and V. C. Gungor, "Packet size optimization in wireless sensor networks for smart grid applications," IEEE Transactions on Industrial Electronics, vol. 64, no. 3, pp. 2392-2401, 2017.

[18] A. Celik and A. E. Kamal, "Multi-objective clustering optimization for multi-channel cooperative spectrum sensing in heterogeneous green crns," IEEE Transactions on cognitive communications and networking, vol. 2, no. 2, pp. 150-161, 2016.

[19] W. Gong, M. Zhang, X. Yang, J. Li, N. Zhang, and K. Long, "WARAS: an adaptive WSN multipath selection model inspired by metabolism behaviors of Escherichia Coli," in Proceedings of the 2015 IEEE/CIC International Conference on IEEE Communications in China (ICCC), Shenzhen, China, November 2015.

[20] J. Li, Q. Yang, P. Gong, and K. S. Kwak, "End-to-end multiservice delivery in selfish wireless networks under distributed node-selfishness management," IEEE Transactions on Communications, vol. 64, no. 3, pp. 1132-1142, 2016.

[21] W. Gong, X. Yang, M. Zhang, and K. Long, "An adaptive path selection model for WSN multipath routing inspired by metabolism behaviors," Science China Information Sciences, vol. 58, no. 10, pp. 1-15, 2015.

[22] P. Mohanty and M. R. Kabat, "Energy efficient reliable multipath data transmission in WSN for healthcare application," International Journal of Wireless Information Networks, vol. 23, no. 2, pp. 162-172, 2016.

[23] R. P. Premanand and A. Rajaram, "Enhanced data accuracy based PATH discovery using backing route selection algorithm in MANET," Peer-to-Peer Networking and Applications, vol. 13, no. 6, pp. 2089-2098, 2020.

[24] A. Rajaram and S. Palaniswami, "Malicious node detection system for mobile adhoc networks," (IJCSIT) International Journal of Computer Science and Information Technologies, vol. 1, no. 2, pp. 77-85, 2010.

[25] S. Palaniswami and A. Rajaram, "An enhanced distributed CertificateAuthority scheme for authentication in mobile ad hoc networks," The International Arab Journal of Information Technology (IAJIT), vol. 9, no. 3, pp. 291-298, 2012. 